\title{
The mean platelet volume on admission predicts unfavorable stroke outcomes in patients treated with IV thrombolysis
}

This article was published in the following Dove Medical Press journal: Clinical Interventions in Aging

Jacek Staszewski'

Aleksandra Pogoda'

Kamila Data'

Klaudia Walczak'

Maciej Nowocień2

Emilia Frankowska ${ }^{2}$

Adam Stępień'

'Clinic of Neurology, Military Institute of Medicine, Warsaw, Poland; ${ }^{2}$ Department of Radiology, Military Institute of Medicine, Warsaw, Poland
Correspondence: Jacek Staszewski Clinic of Neurology, Military Institute of Medicine, Szaserow 128, 04-141 Warsaw, Poland

Tel +4861818419

$\mathrm{Fax}+48228106100$

Email jacekstaszewski@wp.pl
Purpose: The role of biomarkers in the prediction of acute ischemic stroke (AIS) outcome or response to thrombolytic therapy (with recombinant tissue plasminogen activator [rt-PA]) remains limited. The aim of this study was to evaluate whether mean platelet volume (MPV) could predict short-term functional outcome in patients with AIS following rt-PA treatment.

Patients and methods: This was a retrospective analysis of 237 AIS patients (mean age $71.04 \pm 0.8$ years, $50.6 \%$ women) consecutively admitted to a tertiary care center between 2011 and 2015.

Results: The mean MPV in the cohort was $9.8 \pm 0.35 \mathrm{fL}$ (lowest tertile $<7.29 \mathrm{fL}$, median 7.29-8.8 fL, and highest tertile $>8.8 \mathrm{fL}$ ). Patients in the lowest tertile compared to median and highest tertiles were less often dependent (modified Rankin scale $[\mathrm{mRS}] \geq 3$ ) at admission ( $87.2 \%$ vs $96.1 \%$ and $96.1 \%$, respectively, $P=0.04$ ) and less often had a poor stroke outcome (mRS 4-6) at discharge (28.2\% vs 55.3\% and 44.7\%,P<0.01). However, there was no significant difference between tertiles with regard to AIS etiology, CT (Alberta Stroke Program Early CT) score, frequency of stroke due to large artery occlusion, risk of secondary hemorrhage, and early neurologic deterioration. Multivariable analysis after adjustment for confounders showed that patients in the second and third tertiles had a significantly higher risk of poor stroke outcome $(\mathrm{OR}=1.9,95 \% \mathrm{CI}=1.01-4)$, lack of early improvement $(\mathrm{OR}=1.91,95 \% \mathrm{CI}=1.05-3.47)$, lower chance of good outcome ( $\mathrm{mRS} 0-2 ; \mathrm{OR}=0.38,95 \% \mathrm{CI}=0.18-0.78$ ), or minor stroke at discharge $(\mathrm{OR}=0.47,95 \% \mathrm{CI}=0.26-0.84)$. Receiver operating characteristic analysis for prediction of poor stroke outcome showed that the optimal cut-off point of MPV was $8.8 \mathrm{fL}$ (area under the curve 0.586 [0.512-0.659], $P=0.03$ ) with a sensitivity of $82.7 \%$ and a specificity of $43.9 \%$.

Conclusion: Disabling or fatal ischemic stroke in thrombolyzed patients was observed more often in patients with high admission MPV. The prognostic value of MPV was independent of other well-defined individual risk factors.

Keywords: acute ischemic stroke, rt-PA, prognosis, MPV, biomarker, platelet activity, reperfusion

\section{Introduction}

Thrombolytic therapy (with recombinant tissue plasminogen activator [rt-PA]) is an effective treatment for acute ischemic stroke (AIS). The efficacy of rt-PA has been reported to be related to the location of the occlusion, collateral blood supply, and clot-specific factors such as size, composition, and origin of the thrombus; however, reliable predictors of early and late outcomes as well as predictors of intracranial hemorrhage in patients who receive rt-PA in AIS are unknown. ${ }^{1}$ The ideal marker of reperfusion would help predict when standard rt-PA therapy will not lead to prompt artery recanalization; thus, a more specific treatment (ie, mechanical thrombectomy) 
could be immediately adopted in such cases. Unfortunately, clinical and laboratory predictors of failed thrombolysis have not been identified because the mechanisms underlying acute occlusion and the variable response to lytic therapy are still largely unknown.

Hyperactive platelets play a pivotal role in thrombus formation and propagation, leading to acute thrombotic events. ${ }^{2}$ Larger platelets generally contain more dense granules, express more glycoprotein IIb/IIIa receptors, have more thromboxane A2, and aggregate more rapidly with collagen. The mean platelet volume (MPV) is an index of platelet size that correlates with platelet activation, and its precise measurement may provide clues to pathways that can be targeted for interventions aimed at stroke prevention or treatment.

However, most clinical studies on MPV in cardiovascular disease have been performed in patients with coronary artery disease (CAD) in whom MPV was predictive of the infarctrelated artery patency and mortality following acute myocardial infarction. ${ }^{3,4}$ Although patients with AIS differ from those with CAD in their risk factor profile, underlying vessel pathology, and acute treatment, platelets with an increased MPV led to the occurrence of AIS due to carotid stenosis or cardioembolism. ${ }^{5}$ Previously published studies have also shown that an increased MPV was seen in AIS subjects vs controls. ${ }^{6}$ The MPV has been proposed as a predictor of outcome in patients with AIS treated with aspirin; however, the value of MPV in predicting stroke outcome and risk of secondary hemorrhage in patients receiving reperfusion therapy remains unclear. ${ }^{7-9}$

We hypothesized that an elevated MPV level before initiation of rt-PA treatment may be associated with worse AIS outcome than in patients with lower platelet volume. The goal of this study was to investigate whether there is an association between MPV and short-term prognosis in patients with AIS following intravenous (IV) thrombolytic treatment. We also examined the association between baseline MPV measurement and levels of neurologic deficit, early radiologic signs, and AIS etiology.

\section{Patients and methods Study population}

This was a retrospective study of 237 patients consecutively admitted to a tertiary care center over 5 years (01.01.201131.12.2015) due to AIS.

All patients were treated with standard-dose of IV rt-PA according to the National Institute of Neurological Disorders and Stroke schedule and criteria, except that no age restriction was applied and treatment was allowed up to 4.5 hours after onset provided that there were no contraindications in either situation. ${ }^{10}$ Patients treated with intra-arterial thrombolysis or thrombectomy were excluded from this analysis. We also did not include patients with autoimmune disorders, immunosuppression, sepsis, thrombocytopenia, acute leukemia, aplastic anemia, or drug-induced marrow hypoplasia because these diseases could significantly influence MPV and increase the risk of rt-PA complications. All patients received a standardized stroke diagnosis according to the national and international guidelines - this consisted of clinical examinations, cerebral imaging (computed tomography [CT] scans), and carotid/vertebral and transcranial ultrasonography. ${ }^{11}$ We conducted this study in accordance with the Declaration of Helsinki. The electronic database was decoded and patient identification data were scrambled to ensure confidentiality; informed consent was thus exempted. This study was evaluated and approved by the Institutional Review Board of Wojskowy Instytut Medyczny w Warszawie.

Blood samples for MPV and other biochemical markers were drawn from the antecubital vein at baseline within a mean of $14 \pm 7$ minutes of sampling after admission to the Emergency Department (a mean of 2.36+0.33 hours after stroke onset); samples were assessed by a Sysmex XN-1000 hematology autoanalyzer. Samples for MPV were collected in EDTA, and MPV was measured in units of fL (normal range, 7.5-11.5 fL). CT scans were performed immediately at admission and 24 hours after rt-PA or earlier in case of neurologic deterioration (mean $23 \pm 3.5$ hours) to rule out intracranial bleeding. The severity of stroke was assessed immediately at admission, at 1, 24, and 72 hours after rt-PA treatment, and at discharge by a trained and certified senior neurologist in the National Institutes of Health Stroke Scale (NIHSS). ${ }^{12}$ To measure global disability and functional dependence at admission and discharge, we used the modified Rankin scale (mRS) which ranges from 0 (no symptoms) to 6 (death).

\section{Study endpoints}

An excellent functional outcome was defined as mRS discharge score $\leq 1$; a favorable outcome was defined as discharge $\mathrm{mRS}$ score $\leq 2$; and poor stroke outcome was defined if it caused dependence (mRS 3-5) or death during hospitalization. Lack of early improvement was defined as a difference of 3 points or less between the NIHSS score at baseline and at 24 hours. ${ }^{13}$ Early neurologic deterioration (END) was defined as worsening of NIHSS $\geq 4$ points within the first 72 hours after acute stroke compared with 
baseline NIHSS. ${ }^{14}$ Symptomatic intracerebral hemorrhage (sICH) was defined according to the European Cooperative Acute Stroke Study-III criteria (intracerebral hemorrhage combined with clinical deterioration of $\geq 4$ points on NIHSS or death) and it was divided into hemorrhagic infarct type 1 (HI1): small petechiae along the periphery of the infarct; hemorrhagic infarct type 2 (HI2): confluent petechiae within the infarcted area without a space-occupying effect; parenchymal hematoma type 1 (PH1): bleeding $\leq 30 \%$ of the infarcted area with a mild space-occupying effect; and parenchymal hematoma type 2 ( $\mathrm{PH} 2)$ : bleeding $>30 \%$ of the infarcted area with a significant space-occupying effect. ${ }^{15}$ Remote parenchymal hematoma (PHr), solitary or multiple, was defined as hemorrhages that appear in brain regions without visible ischemic damage, remote from the area of ischemia causing the initial stroke symptoms. ${ }^{16}$

Minor ischemic stroke (IS) was defined as a discharge NIHSS score $\leq 4$ and major stroke as NIHSS $>4$ in accordance with previous studies. ${ }^{17}$

\section{Relevant covariates}

The Causative Classification of Stroke (CCS) method was used to classify stroke etiology in accordance with the Stop Stroke Study Trial of Org 10172 in Acute Stroke Treatment criteria. ${ }^{18}$ These criteria integrate the results of clinical evaluations, brain imaging, vascular/heart examinations, and work-up for uncommon causes of stroke. Strokes were categorized into one of the four following categories: cardioembolism, large-artery atherosclerosis, small-vessel occlusion (lacunar stroke), and other (determined etiologies or cryptogenic). If multiple potential causes existed, then the patient was assigned to the undetermined cause group. The CCS has good to excellent intra- and inter-rater reliability. ${ }^{19}$

The time of symptom onset, sex, age, history of antithrombotic medication, hypertension, diabetes mellitus, current smoking, dyslipidemia, atrial fibrillation (AF), CAD, moderate or heavy alcohol consumption ( $\geq 2$ standard alcoholic drinks per day), body mass index, previous stroke or transient ischemic attack, serum glucose, and BP were routinely collected on admission. Hypertension was defined as systolic blood pressure $(\mathrm{SBP}) \geq 140 \mathrm{mmHg}$ or diastolic blood pressure (DBP) $\geq 90 \mathrm{mmHg}$, any use of antihypertensive drugs, or any self-reported history of hypertension. Diabetes mellitus was defined as a fasting glucose level $\geq 7.0 \mathrm{mmol} / \mathrm{L}$, a non-fasting glucose concentration $\geq 11.1 \mathrm{mmol} / \mathrm{L}$, any use of glucose-lowering drugs, or any self-reported history of diabetes. Dyslipidemia was defined as a serum triglyceride (TG) level $\geq 1.7 \mathrm{mmol} / \mathrm{L}$, low-density lipoprotein (LDL) cholesterol $\geq 3.6 \mathrm{mmol} / \mathrm{L}$, high-density lipoprotein (HDL) cholesterol $\leq 1.0 \mathrm{mmol} / \mathrm{L}$, any use of lipid-lowering drugs, or any self-reported history of dyslipidemia. Carotid artery stenosis was recognized if either occlusive or stenotic $(\geq 50 \%$ diameter reduction) vascular disease judged to be due to atherosclerosis was found in the clinically relevant or not relevant extracranial arteries.

The presence of hyperdense middle cerebral artery (MCA) sign on admission CT was recorded - this refers to focal increased density of MCA on CT and is a direct visualization of thromboembolic material within the lumen. The Alberta Stroke Program Early CT Score (ASPECTS) has been used to quantify early ischemic change on baseline CT brain scans of acute stroke patients. ${ }^{20}$ For each of the ten areas, a point was subtracted if there was evidence of early signs of brain ischemia there (eg, reduced attenuation or hypodensity, loss of gray-white matter differentiation, focal swelling, obscuration of the lentiform nucleus, or insular ribbon sign). Old infarcts were ignored. A scan with no ischemia in the MCA territory was scored 10 , and a scan with diffuse involvement of all MCA territories was scored 0 . Intracranial large artery occlusion (LAO) was suspected in patients with baseline NIHSS $\geq 9$ (within 3 hours of stroke onset) or NIHSS $\geq 7$ (within 6 hours) based on the European consensus statement. ${ }^{21}$

\section{Statistical analyses}

All data were first analyzed for normality of distribution using the Kolmogorov-Smirnov test of normality. The results are shown as the mean \pm SD or as counts and percentages. Baseline characteristics of individuals were summarized according to the tertile distribution of MPV. The clinical characteristics of the three groups were compared using oneway ANOVA for continuous variable; the chi-squared test was used to compare the categorical parameters. Comparisons between normally distributed continuous variables were made using ANOVA with post-hoc analysis of least significant difference, and the Kruskal-Wallis test with Bonferoni's post hoc adjustment was used for nonparametric distributed variables. Pearson's correlation coefficients were calculated to evaluate the relationships between MPV and admission clinical and laboratory variables (mRS, NIHSS, SBP, DBP, CT score, morphology, and biochemistry parameters). The distribution of total cholesterol and TG was skewed; hence, we conducted Pearson's linear correlation analysis using log-transformed values instead of the raw data. To measure the strength of the relationship between MPV and discharge NIHSS, mRS, CT ASPECTS score, and other baseline laboratory variables, we used the partial correlation coefficient 
controlled for potential confounders selected based on clinical plausibility and previous literature reviews and known to influence stroke course and linked with high MPV (age, sex, time to rt-PA treatment, and admission NIHSS). ${ }^{22,23} \mathrm{~A}$ multivariable analysis adjusted for the same confounders was performed to assess the effect size and independent relationship between MPV and the stroke outcome. The ORs and 95\% CIs for the upper two tertiles relative to the lowest tertile were calculated. To evaluate the MPV power to predict poor stroke outcome, we also used receiver operating characteristic (ROC). The best cut-off for maximizing sensitivity and specificity according to the Youden index criterion was established. ${ }^{24}$ Statistical significance was set at $P$-value $<0.05$ for all analyses. The statistical analyses were performed with the STATISTICA 17.0 software (StatSoft Inc., Tulsa, OK, USA).

\section{Results}

During the 5 years of this study, 237 patients underwent IV thrombolysis (mean age $71.04 \pm 0.8$ years, $50.6 \%$ women, mean onset to treatment $2.9 \pm 0.1$ hours). Most patients were functionally dependent at admission $(n=214 ; 93 \%)$ and had severe neurologic deficit (mean NIHSS score 11.8 \pm 3.7 ). The admission CT scan showed early stroke signs in 33 patients (14.3\%), and hyperdense MCA sign was found in 35 patients (15.2\%). Symptomatic secondary hemorrhage was found in 27 patients $(11.7 \%)$. The in-hospital mortality rate was $13.5 \%$. Of those who survived, $34 \%(n=67)$ were dependent at discharge. Clinical and imaging characteristics of the cohort are presented in Table 1 .

The mean admission MPV in the cohort was 9.8 $\pm 0.35 \mathrm{fL}$. There were significant differences between MPV tertiles (lowest $<7.29 \mathrm{fL}$, median 7.29-8.8 fL, and highest tertile $>8.8 \mathrm{fL}$ )

Table I Main characteristics of the study population

\begin{tabular}{|c|c|c|c|c|}
\hline Characteristics & $\begin{array}{l}\text { Lowest tertile } \\
(n=78)\end{array}$ & $\begin{array}{l}\text { Median tertile } \\
(n=76)\end{array}$ & $\begin{array}{l}\text { Highest tertile } \\
(n=76)\end{array}$ & $\begin{array}{l}\text { P-value for difference } \\
\text { across tertiles }\end{array}$ \\
\hline MPV range (fL) & $<7.29$ & $7.29-8.8$ & $>8.8$ & \\
\hline \multicolumn{5}{|l|}{ Demographic data } \\
\hline Age (years) & $68.6(12.1)^{* *}$ & $69.8(13.8)$ & $74(1 \mathrm{I} .1)$ & 0.02 \\
\hline Females & $37(47.4)$ & $47(6 \mid .8)^{\#}$ & $32(42.1)$ & 0.04 \\
\hline \multicolumn{5}{|l|}{ Vascular risk factors } \\
\hline Carotid artery stenosis & $21(27)$ & $20(26)$ & $26(34)$ & 0.5 \\
\hline Diabetes & $9(11.5)^{* * *}$ & $18(23.7)$ & $20(26.3)$ & 0.04 \\
\hline Hypertension & $54(69.2)$ & $58(76.3)$ & $57(75)$ & 0.5 \\
\hline Chronic heart failure & $4(5.1)$ & $13(17.1)$ & $10(13.2)$ & 0.04 \\
\hline Coronary artery disease & $20(25.6)$ & $16(21.1)$ & $20(26.3)$ & 0.7 \\
\hline Atrial fibrillation & $14(17.9)^{* * * *}$ & $29(38.2)$ & $29(38.2)$ & $<0.01$ \\
\hline Hyperlipidemia & $27(34.6)$ & $19(25)$ & $19(25)$ & 0.36 \\
\hline IMT (mm) & $1.09(0.24)$ & $1.09(0.29)$ & I.I (0.2) & 0.8 \\
\hline Current smoking & $23(29.5)$ & II (14.5) & $13(17.1)$ & 0.05 \\
\hline Chronic kidney disease & $3(3.8)$ & $5(6.6)$ & $4(5.3)$ & 0.6 \\
\hline Peripheral atherosclerosis & $21(26.9)$ & $20(26.3)$ & $26(34.2)$ & 0.4 \\
\hline Previous stroke/TIA & II (I4.I) & $12(15.8)$ & $10(13.2)$ & 0.8 \\
\hline Previous antithrombotic therapy ${ }^{a}$ & $29(42)$ & $27(38)$ & $33(48)$ & 0.2 \\
\hline \multicolumn{5}{|l|}{ Baseline CT } \\
\hline ASPECTS score & $9.6(I . I)$ & $9.65(0.88)$ & $9.8(0.4)$ & 0.4 \\
\hline ASPECTS $<10$ & $10(14.7)$ & $12(19.4)$ & II (I5.3) & 0.7 \\
\hline Hyperdense MCA sign & $12(15.4)$ & $18(23.7)^{\#}$ & $5(6.6)$ & 0.01 \\
\hline Symptomatic ICH & $8(10.3)$ & $8(10.5)$ & II (I4.5) & 0.05 \\
\hline $\mathrm{HII}$ & $6(7.7)$ & $2(2.6)$ & $3(3.9)$ & \\
\hline $\mathrm{HI} 2$ & 0 & 0 & $4(5.3)$ & \\
\hline $\mathrm{PHI}$ & $2(2.6)$ & $4(5.3)$ & $\mathrm{I}(\mathrm{I} .3)$ & \\
\hline $\mathrm{PH} 2$ & 0 & $2(2.6)$ & $3(3.9)$ & \\
\hline $\mathrm{PHrl}$ & 0 & $\mathrm{I}(\mathrm{I})^{\mathrm{b}}$ & $\mathrm{I}(\mathrm{I})^{\mathrm{b}}$ & \\
\hline $\mathrm{PHr} 2$ & 0 & 0 & $\mathrm{I}(\mathrm{I})^{\mathrm{b}}$ & \\
\hline
\end{tabular}


Table I (Continued)

\begin{tabular}{|c|c|c|c|c|}
\hline Characteristics & $\begin{array}{l}\text { Lowest tertile } \\
(\mathrm{n}=78)\end{array}$ & $\begin{array}{l}\text { Median tertile } \\
(n=76)\end{array}$ & $\begin{array}{l}\text { Highest tertile } \\
(n=76)\end{array}$ & $\begin{array}{l}P \text {-value for difference } \\
\text { across tertiles }\end{array}$ \\
\hline \multicolumn{5}{|l|}{ Stroke etiology } \\
\hline Lacunar stroke & $13(16.7)$ & $12(16)$ & $9(12.2)$ & 0.5 \\
\hline Cardioembolic stroke & $23(29.5)$ & $31(4 I .3)$ & $27(36.5)$ & - \\
\hline Large atherothrombotic stroke & $37(47.4)$ & $24(32)$ & $31(41.9)$ & - \\
\hline Other & $5(6.4)$ & $9(11.8)$ & $9(11.8)$ & - \\
\hline \multicolumn{5}{|c|}{ Baseline biochemical and other variables } \\
\hline Onset to treatment (hours) & $2.78(1.12)$ & $2.85(0.97)$ & $2.95(1.07)$ & 0.6 \\
\hline Onset to blood draw (hours) & $2.2(0.3)$ & $2.5(0.2)$ & $2.4(0.5)$ & 0.5 \\
\hline eGFR $\left(\mathrm{mL} / \mathrm{min} / \mathrm{l} .73 \mathrm{~m}^{2}\right)$ & $85.8(41.2)^{* *}$ & $72.8(31.6)$ & $58.2(24.7)$ & $<0.001$ \\
\hline Creatinine $(\mathrm{mg} / \mathrm{dL})$ & $0.93(0.26)^{* *}$ & $0.97(0.44)$ & $1.19(0.9)$ & 0.01 \\
\hline Blood glucose (mg/dL) & I $19.8(44)$ & I23.I (35.6) & $141.5(77.1)$ & 0.03 \\
\hline Fibrinogen $(\mathrm{mg} / \mathrm{dL})$ & $354.9(109)$ & $367(127)$ & $347(111)$ & 0.6 \\
\hline WBC $\left(10^{3} / \mu \mathrm{L}\right)$ & $8.4(2.9)$ & $9.07(4)$ & $9(3.1)$ & 0.4 \\
\hline $\operatorname{PLT}\left(10^{3} / \mu \mathrm{L}\right)$ & $229.6(68.7)$ & $228.3(76)$ & $218(74.3)$ & 0.5 \\
\hline Total cholesterol (mg/dL) & I82.I $(40.8)^{* * *}$ & $168.3(4 I)$ & I58.I (40.6) & $<0.01$ \\
\hline LDL (mg/dL) & $110.9(39.8)$ & $103(34.4)$ & $102.3(35.5)$ & 0.3 \\
\hline $\mathrm{SBP}(\mathrm{mmHg})$ & $152(25)$ & $154(30)$ & $159(23)$ & 0.2 \\
\hline $\mathrm{DBP}(\mathrm{mmHg})$ & $84.9(13.4)$ & $87.2(18.8)$ & $86.3(14.2)$ & 0.6 \\
\hline \multicolumn{5}{|l|}{ Neurologic status } \\
\hline Admission GCS & I $3.4(2.48)$ & $12.7(2.7)$ & I3.2(2.5) & 0.3 \\
\hline $\mathrm{mRS}$ & $4.05(1.11)^{*}$ & $4.43(0.89)$ & $4.36(0.89)$ & 0.03 \\
\hline Dependence $(\mathrm{mRS} \geq 3)$ & $68(87.2)^{*, * * *}$ & $73(96.1)$ & $73(96.1)$ & 0.04 \\
\hline NIHSS & $10.4(5.1)^{*}$ & $12.5(6.1)$ & $12.4(6)$ & 0.03 \\
\hline Large artery occlusion & $55(70.5)$ & $60(78.9)$ & $56(73.7)$ & 0.4 \\
\hline Symptomatic intracerebral hemorrhage & $8(10.3)$ & $8(10.5)$ & II (14.5) & 0.6 \\
\hline Early neurologic deterioration & II (14.1) & $14(18.4)$ & II (14.5) & 0.7 \\
\hline Discharge mRS & $2.29(1.8)^{* * * *}$ & $3.57(1.9)$ & $3.2(1.9)$ & $<0.001$ \\
\hline NIHSS & $3.9(4.1)^{* * * *}$ & $6.8(5.9)$ & $5.7(5.5)$ & $<0.01$ \\
\hline Favorable outcome (mRS $\leq 2)^{*}$ & $56(71.8)^{* * * *}$ & $34(44.7)$ & $42(55.3)$ & $<0.01$ \\
\hline Poor outcome (mRS $\geq 3$ ) & $22(28.2)^{* * * *}$ & $42(55.3)$ & $34(44.7)$ & $<0.01$ \\
\hline Excellent outcome (mRS $\leq \mathrm{I})$ & $33(42.3)^{* * * *}$ & $16(21.1)$ & $20(26.3)$ & 0.01 \\
\hline Minor stroke $(\mathrm{NIHSS} \leq 4)$ & $51(65.4)^{* * * *}$ & $31(40.8)$ & $38(50)$ & $<0.01$ \\
\hline Death & $6(7.7)$ & $13(17.1)$ & $12(15.8)$ & 0.17 \\
\hline Length of hospitalization (days) & $11.7(4.6)$ & $17.4(16.7)$ & $14.5(11.8)$ & 0.01 \\
\hline Lack of early improvement & $23(29.5)^{* * * *}$ & $34(44.7)$ & $38(50)$ & 0.02 \\
\hline
\end{tabular}

Notes: Values represent the mean $( \pm S D)$ for continuously distributed data or number $(\%)$ for categorical data. P-values were calculated using the ANOVA and chi-squared tests. ${ }^{2}$ Data available from 208 patients ( 68 for first tertile, $7 \mathrm{I}$ for second tertile, and 69 for third tertile). ${ }^{b}$ With concomitant local PH2. $* P<0.05$ between tertiles I and 2 , $* * P<0.05$ between tertiles I and $3, \# P<0.05$ between tertiles 2 and 3 .

Abbreviations: ASPECTS, Alberta Stroke Program Early CT Score; CT, computed tomography; eGFR, estimated glomerular filtration rate; MPV, mean platelet volume; GCS, Glasgow Coma Scale; HI, hemorrhagic infarct; ICH, intracerebral hemorrhage; IMT, intima-media thickness; LDL, low-density lipoprotein; MCA, middle cerebral artery; mRS, modified Rankin Scale; NIHSS, National Institutes of Health Stroke Scale; PH, parenchymal hematoma; PHr, parenchymal hematoma; PLT, platelet; TIA, transient ischemic attack; WBC, white blood cells.

with regard to demographic data, frequency of vascular risk factors, and neurologic status at admission and discharge. Patients in the lowest tertile were younger; had lower prevalence of diabetes, chronic heart failure, and AF; had lower levels of glucose and creatinine; and had higher values for estimated glomerular filtration rate (eGFR) and total cholesterol vs patients in the median or highest MPV tertiles. They had lower admission NIHSS scores, were less often dependent, and rarely experienced early improvement following rt-PA treatment; they had favorable stroke outcome at discharge with a lower mean mRS and NIHSS. There were only few differences between patients in the median 
and highest tertiles - those in the median tertile were more often women and were more likely to have a hyperdense MCA sign. However, there were no significant differences between tertiles with regard to AIS etiology, frequency of carotid artery stenosis, antithrombotic treatment, CT ASPECTS score, frequency of stroke due to LAO, and risk of END. Also, the difference between the risk of sICH and the subtype distribution of all hemorrhage types did not reach statistical significance.

There was a weak linear positive correlation between MPV and age, creatinine, glucose levels, SBP at admission, and $\mathrm{mRS}$ at discharge. There was a negative correlation between MPV and eGFR and total cholesterol at admission (Table 2). Similarly, after correcting for confounders, MPV correlated with eGFR, creatinine, glucose, total cholesterol, and discharge $\mathrm{mRS}$. Among the variables available on admission, only age $(\mathrm{OR}=1.003,95 \% \mathrm{CI}=1.01-1.05)$, diabetes $(\mathrm{OR}=1.5,95 \% \mathrm{CI}=1.2-1.9), \mathrm{AF}(\mathrm{OR}=1.4,95 \% \mathrm{CI}=1.2-1.6)$, cardioembolic stroke $(\mathrm{OR}=1.8,95 \% \mathrm{CI}=1.4-4.2), \mathrm{mRS}$ $(\mathrm{OR}=6.5,95 \% \mathrm{CI}=3.5-11.7)$, and NIHSS (OR $=1.2,95 \%$ $\mathrm{CI}=1.1-1.3)$ were significantly related to poor outcome in univariate analysis. Multivariable analysis after adjustment for age, sex, delay to rt-PA treatment, and baseline NIHSS showed significant correlations between MPV and clinical outcomes

Table 2 Correlation and partial correlation analysis between tertiles of MPV as well as clinical and biochemical parameters

\begin{tabular}{|l|l|l|l|l|}
\hline Variables & $\boldsymbol{r}$ & $\boldsymbol{P}$-value & $\boldsymbol{r}^{\mathbf{a}}$ & $\boldsymbol{P}_{\text {-value }}$ \\
\hline Age & 0.18 & $<0.01$ & - & - \\
\hline Onset to treatment & 0.05 & 0.14 & - & - \\
\hline Admission NIHSS & 0.1 & 0.05 & - & - \\
\hline Admission mRS & 0.1 & 0.07 & 0.19 & 0.06 \\
\hline eGFR & -0.29 & $<0.00 \mathrm{I}$ & -0.2 & 0.04 \\
\hline Creatinine & 0.15 & $<0.01$ & 0.21 & 0.04 \\
\hline Glucose & 0.16 & 0.01 & 0.24 & 0.02 \\
\hline Fibrinogen & -0.06 & 0.2 & 0.01 & 0.8 \\
\hline WBC & 0.06 & 0.18 & 0.17 & 0.09 \\
\hline PLT & -0.08 & 0.1 & -0.1 & 0.3 \\
\hline Total cholesterol & -0.17 & $<0.01$ & -0.2 & 0.04 \\
\hline LDL & -0.05 & 0.3 & -0.12 & 0.1 \\
\hline SBP & 0.11 & 0.03 & 0.05 & 0.6 \\
\hline DBP & 0.04 & 0.4 & 0.04 & 0.6 \\
\hline CT ASPECTS score & 0.01 & 0.9 & 0.07 & 0.5 \\
\hline Discharge mRS & 0.16 & $<0.01$ & 0.29 & $<0.01$ \\
\hline Discharge NIHSS & 0.1 & 0.06 & 0.14 & 0.18 \\
\hline
\end{tabular}

Notes: Coefficients $(r)$ and P-values are calculated using the Pearson's correlation

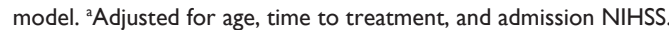

Abbreviations: ASPECTS, Alberta Stroke Program Early CT Score; CT, computed tomography; eGFR, estimated glomerular filtration rate; LDL, low-density lipoprotein; MPV, mean platelet volume; mRS, modified Rankin Scale; NIHSS, National Institutes of Health Stroke Scale; PLT, platelet; WBC, white blood cells.
(Figure 1). Patients in the second and third tertiles had a significantly higher risk of poor stroke outcome $(\mathrm{OR}=1.9,95 \%$ $\mathrm{CI}=1.01-4.2)$, lack of early improvement $(\mathrm{OR}=1.9,95 \%$ $\mathrm{CI}=1.04-3.5)$, lower chance of favorable outcome $(\mathrm{OR}=0.37$, $95 \% \mathrm{CI}=0.18-0.75)$, or minor stroke at discharge $(\mathrm{OR}=0.57$, $95 \% \mathrm{CI}=0.27-0.99)$ vs patients in the first tertile. They also had a lower chance of excellent stroke outcome $(\mathrm{OR}=0.52$, 95\% CI $=0.26-1.02$ ). The risk of END, sICH, or death was similar across all tertiles of MPV. Lower chance of favorable outcome and lack of early improvement were significantly related to second and third MPV tertiles after adding other confounders also (stroke etiology, diabetes, and AF) into the multivariate model. ROC analysis for prediction of poor stroke outcome showed that the optimal cut-off point of MPV was $8.8 \mathrm{fL}$ (area under the curve 0.586 [0.512-0.659], $P=0.03$ ) with $82.7 \%$ sensitivity and $43.9 \%$ specificity (Figure 2 ).

\section{Discussion}

This study found that MPV may be an early and easily measured predictor of prognosis in AIS patients treated with rt-PA. We demonstrated that patients in the medium and upper tertiles of MPV had a higher risk of poor stroke outcome and a lower chance of excellent outcome following rt-PA treatment. Moreover, patients in the lowest tertile of MPV were less often dependent at admission and scored lower on admission mRS and NIHSS. However, the MPV was not predictive of stroke etiology, LAO, early stroke radiological signs, and risk of complications (sICH or END) following rt-PA therapy. As MPV has been considered to reflect platelet activity, our findings provide further evidence that platelet activation plays a role in the course of hyperacute AIS following rt-PA treatment.

Thrombolytic therapy with rt-PA is a standard treatment for patients with AIS, and it has a Class $1 \mathrm{~A}$ recommendation according to current guidelines. ${ }^{25}$ In the last decade, there has been an increased focus on early identification of accurate markers of thrombolytic success and identification for patients at risk of developing complications following rt-PA therapy. Such markers could help to stratify which patients are likely to benefit from selective use of additional interventions, for example, mechanical thrombectomy. There are several well-established predictors of poor outcome following rt-PA therapy, for example, long onset to treatment time, elderly age, high admission NIHSS score, high blood glucose, and early hypodensity area on neuroimaging. ${ }^{26}$ Other parameters, for example, high SBP, AF, weight, and severe stroke at admission, increase the risk of intracranial hemorrhage. ${ }^{27,28}$ Most of these factors, however, provide only an approximation of the efficacy and safety of rt-PA 


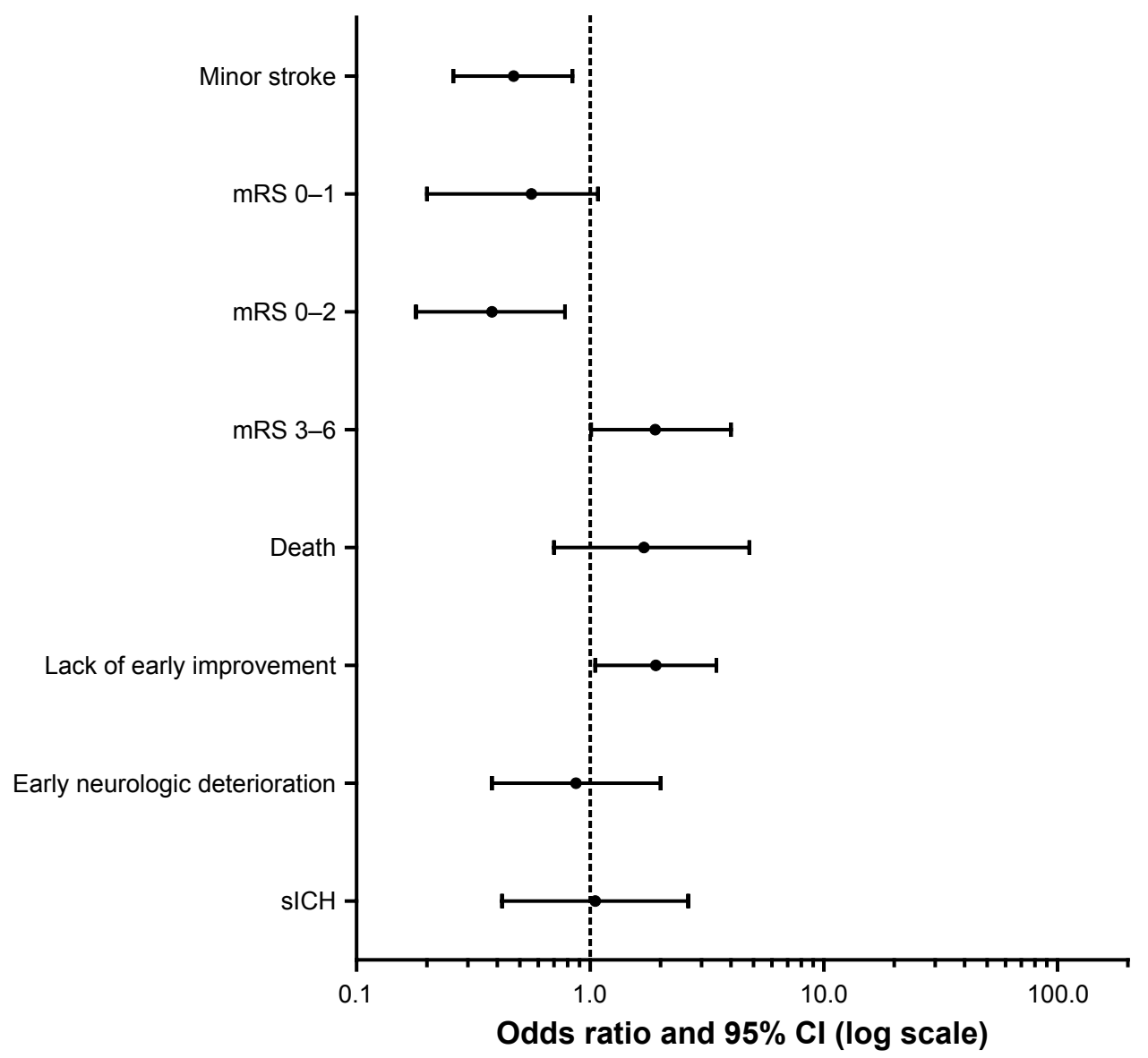

Figure I Associations between tertiles of MPV and clinical outcomes. ${ }^{a}$

Note: aAdjusted by age, sex, onset to treatment, and baseline NIHSS.

Abbreviations: MPV, mean platelet volume; mRS, modified Rankin Scale; NIHSS, National Institutes of Health Stroke Scale; sICH, symptomatic intracerebral hemorrhage.

therapy and are not predictive of outcome for an individual patient - there remains a substantial need to develop a simple and quick tool that could help clinicians predict stroke outcome at bedside.

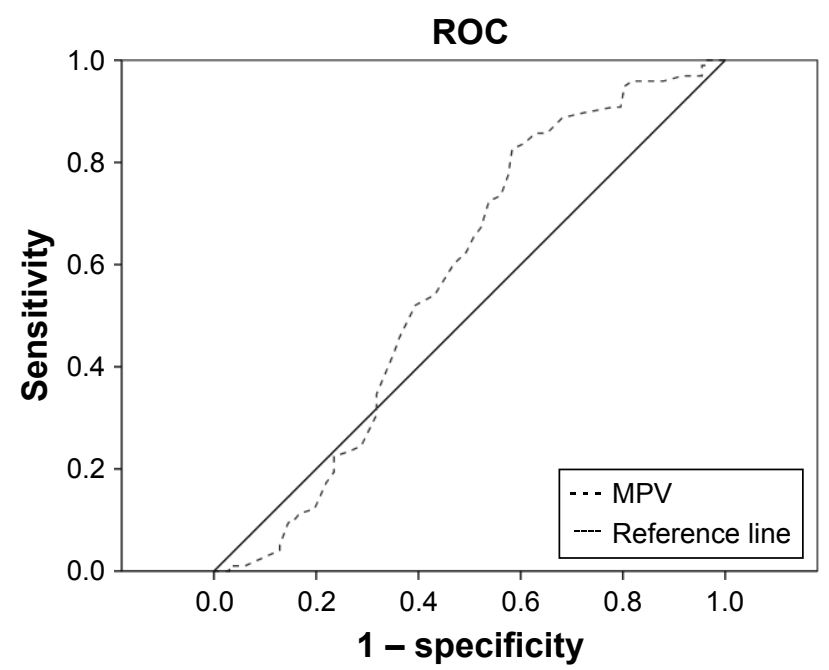

Figure 2 ROC curve for baseline MPV.

Abbreviations: MPV, mean platelet volume; ROC, receiver operating characteristic.
Our results suggest that patients within middle or high tertiles of MPV on admission represent the highest risk for larger infarcts on admission and worse outcome following rt-PA therapy; however, the MPV was not predictive of hemorrhagic complications or early neurologic deterioration. The relationship between MPV and poor AIS outcome has been previously described, but only a few studies have evaluated the prognostic value of MPV in patients treated with rt-PA. ${ }^{29-32}$ An association between lower MPV and improvement in the NIHSS and mRS scores at 24 hours and 3 months after discharge from the hospital was recently shown by İnanç et al, ${ }^{9}$ but no correlations were found with neurologic deterioration, and the results were not controlled for important confounders such as admission stroke severity and timing of MPV measurement after stroke. ${ }^{8}$ du et al demonstrated that elevated admission MPV was correlated with poor prognosis at 30 days, but the association was not significant after adjusting for pretreatment NIHSS; other confounders were not assessed, and the number of patients who received rt-PA in that cohort was not stated. ${ }^{33}$ Most studies on patients who received antiplatelet therapy 
demonstrated the association between MPV and worse functional status or higher mRS scores at 24 hours and at 3 months post-admission. ${ }^{34,35}$ Muscari et al revealed that patients with MPV in the highest quantile had $>2$-fold higher risk of in-hospital dependence or death than groups with MPV in the lowest quantile. ${ }^{36}$ Contrasting results have also been published. Ciancarelli et al found no relationship between MPV values at baseline and NIHSS and mRS at 2 months, but they revealed that MPV decreases following 8 weeks of neurorehabilitation. ${ }^{37}$ Ntaios et al described no significant difference in the frequency of minor strokes and good functional outcome at 3 and 12 months across MPV quartiles. ${ }^{38}$ The exact mechanism by which MPV might influence stroke outcome is unknown and multiple mechanisms may be involved. MPV is associated with platelet activity, aggregation, thromboxane B2 release, and increased expression of the platelet adhesion molecule glycoprotein IIb/IIIa, and several clinical studies reported that an elevated MPV is associated with different thromboembolic diseases.

Some studies revealed that high MPV results from combined stroke risk factors and simply reflects an increased platelet activity representing the pre-stroke status, decreased HDL levels, hypertension, and advanced age of patients; it might also represent inflammatory status. ${ }^{34,39}$ Progressive impairment of endothelial function plays a crucial role in the aging process, and therefore leads to the production of large platelets. An increase in MPV has also been reported to be associated with race, sex, obesity, smoking, and glucose and creatinine levels, with an inverse correlation with eGFR; this is similar to our data. ${ }^{40-42}$ An inverse relationship normally exists between MPV and the platelet count within the normal range - this probably contributes to the maintenance of normal hemostatic function. Moreover, we found that total cholesterol levels were independently associated with MPV - this agrees with some studies that demonstrated binding of LDL, very low-density lipoprotein, and LDL to the platelet surface; in vitro, LDL stimulated platelet aggregation similar to common platelet agonists. ${ }^{43}$

There are multiple studies with conflicting results describing relations between MPV and symptomatic carotid artery stenosis or artery plaques. ${ }^{44-46}$ However, we found no differences in MPV tertiles between patients with significant carotid artery stenosis or between patients with different stroke etiology; these data correspond to O'Malley et al who reported high values of MPV in all subtypes of IS. ${ }^{47}$ Although MPV was higher in patients with AF, this agreed with previous studies that demonstrated the association between MPV and markers of left atrial stasis in patients with $\mathrm{AF}{ }^{48}$
Our data showed that larger MPV predicted poor stroke outcome and more severe stroke at admission, and these results confirm previous reports showing the association between MPV and a larger admission infarct volume on CT brain scans. ${ }^{49}$ Activated platelets are probably implicated both in the pathophysiology of stroke and stroke development. They play an important role in plaque destabilization, thrombus formation, and progression. ${ }^{50}$ Platelets express and secrete many substances that are crucial mediators of prothrombotic state and atherosclerosis, linking hemostasis and immunity in both directions. Larger platelets contain more thrombotic substances (platelet factor 4, P-selectin, platelet-derived growth factor) and show decreased inhibition of aggregation by prostacyclin and greater aggregability in response to ADP in vitro. ${ }^{51}$ They can aggravate clot formation through the platelet/fibrinogen interactions and regulate inflammatory reactions and endothelial permeability, which may influence both short- and long-term course of stroke..$^{52,53}$ The platelets' involvement in the interplay between thrombosis, inflammation, and hemostasis is likely complex, yet extremely important in each disease process. Several of the proinflammatory mediators implicated in IS (eg, IL-6, IL-8, IL-1 $\beta$, and monocyte chemotactic protein 1) induce platelet activation (and thus, MPV) and platelet-leukocyte aggregation, and they promote a variety of responses that favor coagulation and thrombosis. ${ }^{54,55}$ These interactions are critical for hemostasis and the function of endothelial cells, and they have been suggested as important factors contributing to plaque rupture, thrombus formation, and unfavorable prognosis after AIS. ${ }^{56,57}$

However, it is not clear whether MPV could be increased secondary to the extent of acute brain injury after IS. Here, both admission and discharge mRS and NIHSS scores were the highest in medium and upper tertiles of MPV; however, MPV remained significantly correlated with discharge neurologic status also after adjustment for admission $\mathrm{mRS}$, and it did not differ between patients with early CT signs of brain ischemia. This could also suggest that activated platelets may contribute to reperfusion injury and "no reflow" phenomenon after AIS and play a more important role in collateral circulation and in microvessels, which has been shown in animal models. ${ }^{58}$

The MPV is widely available, cheap, and available before rt-PA therapy as a part of morphology assessment; however, numerous other biomarkers have been studied to improve early diagnosis or predict clinical outcome and response to stroke therapy, but currently none of these has sufficient accuracy for routine clinical practice. Many studies revealed that CRP (C-reactive protein) correlated with the infarct size, 
risk of hemorrhage, or worse prognosis; but this association was weak or insignificant after adjustment for age or stroke severity. ${ }^{59-61}$ Studies also showed a decrease in fibrinogen level following rt-PA therapy, which was associated with the risk of sICH and poor prognosis at 3 months. ${ }^{62}$ Also, higher matrix metallopeptidase-9 (MMP-9) and copeptin levels were shown to be associated with infarct volume, stroke severity, and clinical outcomes. ${ }^{63-65}$ Other studies demonstrated that elevated brain natriuretic protein and D-dimer levels were associated with increased stroke mortality, but other studies found no such association. ${ }^{66-69}$ Furthermore, MMP-9 and S-100B concentrations were related to the risk of hemorrhagic complications after thrombolysis; however, their use is still limited by a lack of availability and high cost. ${ }^{70}$

\section{Limitations}

This study has several limitations. It was limited by the absence of a control group, and its retrospective and singlecenter design may have led to bias; thus, our findings should be considered as hypothesis generating at best. We were also unable to explore the exact mechanism of MPV in the course of stroke. Furthermore, it is unclear how our findings might impact treatment because our analyses were limited to short-term in-hospital outcomes, while prognostic stroke studies used the mRS as the primary outcome measure after 3 months. ${ }^{71}$ Prospective studies with long-term follow-up data or imaging approaches in both the hyperacute and subacute stages would provide more reliable findings. Another limitation is that we did not have data on the pre-stroke use of antithrombotic drugs from all patients or lipidlowering drugs, and we did not routinely perform exams to assess leptomeningeal collateral circulation, reperfusion/ recanalization, and infarct volume, which are important variables influencing short-term outcome in patients treated with IV rt-PA. An additional important limitation is that the normal ranges for the MPV can be influenced by factors such as the anticoagulant used in the collection tube and the delay in time from sampling to analysis; however, this delay was very short in our cohort and all measurements were performed within 30 minutes after collection, using the same device. However, it should be stressed that different automated cell counters and platelet-counting methods as well as MPV measurements at different time intervals from stroke onset are important factors underlying different results and contradictions between studies. ${ }^{72}$

Strengths of the study include the large sample size and focusing on different stroke outcomes. To the best of our knowledge, the association between MPV and the course of stroke patients receiving rt-PA has not been previously studied in a Polish population. We found that high MPV values on admission were closely associated with stroke outcome at discharge in patients who received rt-PA therapy in AIS. In conjunction with other laboratory tests or radiological data, MPV measurements that are already available in routine laboratories may represent one of the easiest measurements to be used as a prognostic marker and help with decision making and selecting optimal treatment strategies without adding economic burden. The combination of MPV with stroke scores including vascular risk factors as well as clinical and imaging data could increase the predictive value for early risk of recurrence, early death, and progressive stroke after IS. However, the methodological problems involved in obtaining an accurate MPV result must be considered and the results should be carefully standardized. ${ }^{73}$ Our results should be verified in a prospective study with a larger sample size to clarify the role of MPV during AIS and how patients with high MPV should be handled regarding optimal stroke management including reperfusion therapy. It is important to note that MPV could give some information regarding the thrombocytes' activation, but the final evidence will be the functional tests for platelet activity; therefore, further investigations are required to elucidate the precise mechanisms through which circulating platelets affect the prognosis in AIS.

\section{Conclusion}

Disabling or fatal IS in thrombolyzed patients was observed more often in patients with high admission MPV. The prognostic value of MPV was independent of other welldefined individual risk factors. It is not clear if MPV has a direct contribution to AIS outcomes; therefore, further studies are needed to explain the underlying mechanism.

\section{Disclosure}

The authors report no conflicts of interest in this work.

\section{References}

1. Molina CA, Montaner J, Arenillas JF, Ribo M, Rubiera M, Alvarez-Sabín J. Differential pattern of tissue plasminogen activator-induced proximal middle cerebral artery recanalization among stroke subtypes. Stroke. 2004;35(2):486-490.

2. Ross R. Atherosclerosis - an inflammatory disease. NEngl J Med. 1999; 340(2):115-126.

3. Duygu H, Turkoglu C, Kirilmaz B, Turk U. Effect of mean platelet volume on postintervention coronary blood flow in patients with chronic stable angina pectoris. J Invasive Cardiol. 2008;20(3):120-124.

4. Chu SG, Becker RC, Berger PB, et al. Mean platelet volume as a predictor of cardiovascular risk: a systematic review and meta-analysis. J Thromb Haemost. 2010;8(1):148-156.

5. Senaran H, Ileri M, Altinbaş A, et al. Thrombopoietin and mean platelet volume in coronary artery disease. Clin Cardiol. 2001;24(5) 405-408. 
6. Chen Y, Xiao Y, Lin Z, et al. The role of circulating platelets microparticles and platelet parameters in acute ischemic stroke patients. J Stroke Cerebrovasc Dis. 2015;24(10):2313-2320.

7. Greisenegger S, Endler G, Hsieh K, Tentschert S, Mannhalter C, Lalouschek W. Is elevated mean platelet volume associated with a worse outcome in patients with acute ischemic cerebrovascular events? Stroke. 2004;35(7):1688-1691.

8. Oji S, Tomohisa D, Hara W, et al. Mean platelet volume is associated with early neurological deterioration in patients with branch atheromatous disease: involvement of platelet activation. J Stroke Cerebrovasc Dis. 2018;27(6):1624-1631.

9. Inanç Y, Giray S, Inanç Y, Yusuf I, Semih G, Yilmaz I. Mean platelet volume, C-reactive protein, and prognosis in patients with acute ischemic stroke following intravenous thrombolytic treatment. Med Sci Monit. 2018;24:3782-3788.

10. Postępowanie W udarze mózgu: wytyczne Grupy Ekspertów Sekcji Chorób Naczyniowych Polskiego Towarzystwa Neurologicznego. Neurol i Neurochir Pol. 2012;46(Suppl 1).

11. Kernan WN, Ovbiagele B, Black HR, et al. Guidelines for the prevention of stroke in patients with stroke and transient ischemic attack: a guideline for healthcare professionals from the American Heart Association/ American Stroke Association. Stroke. 2014;45(7):2160-2236.

12. Frankel MR, Morgenstern LB, Kwiatkowski T, et al. Predicting prognosis after stroke: a placebo group analysis from the National Institute of Neurological Disorders and Stroke rt-PA Stroke Trial. Neurology. 2000;55(7):952-959.

13. Saposnik G, Young B, Silver B, et al. Lack of improvement in patients with acute stroke after treatment with thrombolytic therapy: predictors and association with outcome. JAMA. 2004;292(15):1839-1844.

14. Awadh M, MacDougall N, Santosh C, Teasdale E, Baird T, Muir KW. Early recurrent ischemic stroke complicating intravenous thrombolysis for stroke: incidence and association with atrial fibrillation. Stroke. 2010;41(9):1990-1995.

15. Hacke W, Kaste M, Fieschi C, et al. Intravenous thrombolysis with recombinant tissue plasminogen activator for acute hemispheric stroke: the European cooperative acute Stroke Study (ECASS). $J$ Am Med Assoc. 1995;274:1017-1025.

16. Trouillas P, von Kummer R. Classification and pathogenesis of cerebral hemorrhages after thrombolysis in ischemic stroke. Stroke. 2006;37(2):556-561.

17. Breuer L, Blinzler C, Huttner HB, Kiphuth IC, Schwab S, Köhrmann M. Off-label thrombolysis for acute ischemic stroke: rate, clinical outcome and safety are influenced by the definition of 'minor stroke'. Cerebrovasc Dis. 2011;32(2):177-185.

18. Ay H, Furie KL, Singhal A, Smith WS, Sorensen AG, Koroshetz WJ. An evidence-based causative classification system for acute ischemic stroke. Ann Neurol. 2005;58(5):688-697.

19. Arsava EM, Ballabio E, Benner T, et al. The causative classification of stroke system: an international reliability and optimization study. Neurology. 2010;75(14):1277-1284.

20. Pexman JH, Barber PA, Hill MD, et al. Use of the Alberta Stroke Program Early CT Score (ASPECTS) for assessing CT scans in patients with acute stroke. AJNR Am J Neuroradiol. 2001;22(8):1534-1542.

21. Wahlgren N, Moreira T, Michel P, et al. Mechanical thrombectomy in acute ischemic stroke: consensus statement by ESO-Karolinska stroke update 2014/2015, supported by ESO, ESMINT, ESNR and EAN. Int J Stroke. 2016;11(1):134-147.

22. Caso V, Michel P. Thrombolysis in acute stroke. Lancet. 2015; 385(9976):1395-1396.

23. Mayda-Domaç F, Misirli H, Yilmaz M. Prognostic role of mean platelet volume and platelet count in ischemic and hemorrhagic stroke. J Stroke Cerebrovasc Dis. 2010;19(1):66-72.

24. Fluss R, Faraggi D, Reiser B. Estimation of the Youden index and its associated cutoff point. Biom J. 2005;47(4):458-472.

25. Powers WJ, Rabinstein AA, Ackerson T, et al. Guidelines for the early management of patients with acute ischemic stroke: a guideline for healthcare professionals from the American Heart Association/ American Stroke Association. Stroke. 2018;2018(49):e46-e110.
26. Emberson J, Lees KR, Lyden P, et al. Effect of treatment delay, age, and stroke severity on the effects of intravenous thrombolysis with alteplase for acute ischaemic stroke: a meta-analysis of individual patient data from randomised trials. Lancet. 2014;384(9958):1929-1935.

27. Wahlgren N, Ahmed N, Eriksson N, et al. Multivariable analysis of outcome predictors and adjustment of main outcome results to baseline data profile in randomized controlled trials: Safe Implementation of Thrombolysis in Stroke-MOnitoring study (SITS-MOST). Stroke. 2008; 39(12):3316-3322.

28. Whiteley WN, Slot KB, Fernandes P, Sandercock P, Wardlaw J. Risk factors for intracranial hemorrhage in acute ischemic stroke patients treated with recombinant tissue plasminogen activator: a systematic review and meta-analysis of 55 studies. Stroke. 2012;43(11):2904-2909.

29. Arikanoglu A, Yucel Y, Acar A, Cevik MU, Akil E, Varol S. The relationship of the mean platelet volume and C-reactive protein levels with mortality in ischemic stroke patients. Eur Rev Med Pharmacol Sci. 2013;17(13):1774-1777.

30. Mayda-Domaç F, Misirli H, Yilmaz M. Prognostic role of mean platelet volume and platelet count in ischemic and hemorrhagic stroke. J Stroke Cerebrovasc Dis. 2010;19(1):66-72.

31. Greisenegger S, Endler G, Hsieh K, Tentschert S, Mannhalter C, Lalouschek W. Is elevated mean platelet volume associated with a worse outcome in patients with acute ischemic cerebrovascular events? Stroke. 2004;35(7):1688-1691.

32. Butterworth RJ, Bath PM. The relationship between mean platelet volume, stroke subtype and clinical outcome. Platelets. 1998;9(6):359-364.

33. Du J, Wang Q, He B, et al. Association of mean platelet volume and platelet count with the development and prognosis of ischemic and hemorrhagic stroke. Int J Lab Hematol. 2016;38(3):233-239.

34. Elsayed AM, Mohamed GA. Mean platelet volume and mean platelet volume/platelet count ratio as a risk stratification tool in the assessment of severity of acute ischemic stroke. Alexandria J Med. 2017; 53(1):67-70.

35. Arévalo-Lorido JC, Carretero-Gómez J, Álvarez-Oliva A, GutiérrezMontaño C, Fernández-Recio JM, Najarro-Díez F. Mean platelet volume in acute phase of ischemic stroke, as predictor of mortality and functional outcome after 1 year. J Stroke Cerebrovasc Dis. 2013;22(4): 297-303.

36. Muscari A, Puddu GM, Cenni A, et al. Mean platelet volume (MPV) increase during acute non-lacunar ischemic strokes. Thromb Res. 2009;123(4):587-591.

37. Ciancarelli I, De Amicis D, Di Massimo C, Pistarini C, Ciancarelli MG. Mean platelet volume during ischemic stroke is a potential proinflammatory biomarker in the acute phase and during neurorehabilitation not directly linked to clinical outcome. Curr Neurovasc Res. 2016;13(3):177-183.

38. Ntaios G, Gurer O, Faouzi M, Aubert C, Michel P. Mean platelet volume in the early phase of acute ischemic stroke is not associated with severity or functional outcome. Cerebrovasc Dis. 2010;29(5):484-489.

39. Kamisli O, Kamisli S, Kablan Y, Gonullu S, Ozcan C. The prognostic value of an increased mean platelet volume and platelet distribution width in the early phase of cerebral venous sinus thrombosis. Clin Appl Thromb Hemost. 2013;19(1):29-32.

40. Cao X, Xie X, Zhou J, Yang P, Wang Y, Chen Z. Increased platelet volume in a general population with prehypertension: a cross-sectional study of 80545 participants from China. Hypertens Res. 2012;35(9): 903-908.

41. Verdoia M, Barbieri L, Schaffer A, et al. Impact of renal function on mean platelet volume and its relationship with coronary artery disease: a single-centre cohort study. Thromb Res. 2016;141:139-144.

42. Keating FK, Sobel BE, Schneider DJ. Effects of increased concentrations of glucose on platelet reactivity in healthy subjects and in patients with and without diabetes mellitus. Am J Cardiol. 2003;92(11):1362-1365.

43. Pedreño J, Hurt-Camejo E, Wiklund O, Badimón L, Masana L. Lowdensity lipoprotein (LDL) binds to a G-protein coupled receptor in human platelets. Evidence that the proaggregatory effect induced by LDL is modulated by down-regulation of binding sites and desensitization of its mediated signaling. Atherosclerosis. 2001;155(1):99-112. 
44. Arévalo-Lorido JC, Carretero-Gómez J, Villar-Vaca P. Mean platelet volume predicting carotid atherosclerosis in atherothrombotic ischemic stroke. Ir J Med Sci. 2012;181(2):179-183.

45. Oz II, Yucel M, Bilici M, et al. Is mean platelet volume a reliable marker to predict ischemic stroke in the follow-up of patients with carotid stenosis? J Stroke Cerebrovasc Dis. 2016;25(2):404- 409.

46. Koklu E, Yuksel IO, Arslan S, et al. Predictors of symptom development in intermediate carotid artery stenosis: mean platelet volume and platelet distribution width. Angiology. 2016;67(7):622-629.

47. O’Malley T, Langhorne P, Elton RA, Stewart C. Platelet size in stroke patients. Stroke. 1995;26(6):995-999.

48. Colkesen Y, Acil T, Abayli B, et al. Mean platelet volume is elevated during paroxysmal atrial fibrillation: a marker of increased platelet activation? Blood Coagul Fibrinolysis. 2008;19(5):411-414.

49. Pikija S, Cvetko D, Hajduk M, Trkulja V. Higher mean platelet volume determined shortly after the symptom onset in acute ischemic stroke patients is associated with a larger infarct volume on CT brain scans and with worse clinical outcome. Clin Neurol Neurosurg. 2009;111(7): $568-573$.

50. Xu XR, Carrim N, Neves MA, et al. Platelets and platelet adhesion molecules: novel mechanisms of thrombosis and anti-thrombotic therapies. Thromb J. 2016;14(Suppl 1):29.

51. Viera-de-Abreu A, Rondina MT, Weyrich AS, Zimmermann GA. The role of platelets in disease: inflammation. In: Michelson AD, editor. Platelets. 3rd ed. New York: Elsevier Inc.; 2013:733-766.

52. del Zoppo GJ. The role of platelets in disease: central nervous system ischemia. In: Michelson AD, editor. Platelets. 3rd ed. Elsevier Inc.; 2013:669-698.

53. Ho-Tin-Noé B, Demers M, Wagner DD. How platelets safeguard vascular integrity. J Thromb Haemost. 2011;9(Suppl 1):56-65.

54. Franks ZG, Campbell RA, Weyrich AS, Rondina MT. Plateletleukocyte interactions link inflammatory and thromboembolic events in ischemic stroke. Ann N Y Acad Sci. 2010;1207(1):11-17.

55. Tang YH, Vital S, Russell J, Seifert H, Granger DN. Interleukin-6 mediates enhanced thrombus development in cerebral arterioles following a brief period of focal brain ischemia. Exp Neurol. 2015;271:351-357.

56. Welsh P, Lowe GD, Chalmers J, et al. Associations of proinflammatory cytokines with the risk of recurrent stroke. Stroke. 2008;39(8): 2226-2230.

57. Campbell DJ, Woodward M, Chalmers JP, et al. Soluble vascular cell adhesion molecule 1 and N-terminal pro-B-type natriuretic peptide in predicting ischemic stroke in patients with cerebrovascular disease. Arch Neurol. 2006;63(1):60-65.

58. Nieswandt B, Pleines I, Bender M. Platelet adhesion and activation mechanisms in arterial thrombosis and ischaemic stroke. J Thromb Haemost. 2011;9 (Suppl 1):92-104.
59. Karlinski M, Bembenek J, Grabska K, et al. Routine serum C-reactive protein and stroke outcome after intravenous thrombolysis. Acta Neurol Scand. 2014;130(5):305-311.

60. Elkind MS, Luna JM, Moon YP, et al. High-sensitivity C-reactive protein predicts mortality but not stroke: the Northern Manhattan Study. Neurology. 2009;73(16):1300-1307.

61. Di Napoli M, Papa F, Bocola V. C-reactive protein in ischemic stroke: an independent prognostic factor. Stroke. 2001;32(4):917.

62. Matosevic B, Knoflach M, Werner P, et al. Fibrinogen degradation coagulopathy and bleeding complications after stroke thrombolysis. Neurology. 2013;80(13):1216-1224.

63. Montaner J, Molina CA, Monasterio J, et al. Matrix metalloproteinase-9 pretreatment level predicts intracranial hemorrhagic complications after thrombolysis in human stroke. Circulation. 2003;107(4):598-603.

64. Ramos-Fernandez M, Bellolio MF, Stead LG. Matrix metalloproteinase-9 as a marker for acute ischemic stroke: a systematic review. J Stroke Cerebrovasc Dis. 2011;20(1):47-54.

65. Katan M, Fluri F, Morgenthaler NG, et al. Copeptin: a novel, independent prognostic marker in patients with ischemic stroke. Ann Neurol. 2009; 66(6):799-808.

66. Garcia-Berrocoso T, Giralt D, Bustamante A, et al. B-type natriuretic peptides and mortality after stroke: a systematic review and metaanalysis. Neurology. 2013;81(23):1976-1985.

67. Barber M, Langhorne P, Rumley A, Lowe GD, Stott DJ. Hemostatic function and progressing ischemic stroke: D-dimer predicts early clinical progression. Stroke. 2004;35(6):1421.

68 del Zoppo GJ, Levy DE, Wasiewski WW, et al. Hyperfibrinogenemia and functional outcome from acute ischemic stroke. Stroke. 2009; 40(5):1687-1691.

69. Welsh P, Barber M, Langhorne P, et al. Associations of inflammatory and haemostatic biomarkers with poor outcome in acute ischaemic stroke. Cerebrovasc Dis. 2009;27(3):247-253.

70. Foerch C, Wunderlich MT, Dvorak F, et al. Elevated serum S100B levels indicate a higher risk of hemorrhagic transformation after thrombolytic therapy in acute stroke. Stroke. 2007;38(9):2491-2495.

71. König IR, Ziegler A, Bluhmki E, et al. Predicting long-term outcome after acute ischemic stroke: a simple index works in patients from controlled clinical trials. Stroke. 2008;39(6):1821-1826.

72. Bowles KM, Cooke LJ, Richards EM, Baglin TP. Platelet size has diagnostic predictive value in patients with thrombocytopenia. Clin Lab Haematol. 2005;27(6):370-373.

73. Machin SJ, Briggs C. Mean platelet volume: a quick, Easy determinant of thrombotic risk? J Thromb Haemost. 2010;8(1):146-147.
Clinical Interventions in Aging

\section{Publish your work in this journal}

Clinical Interventions in Aging is an international, peer-reviewed journal focusing on evidence-based reports on the value or lack thereof of treatments intended to prevent or delay the onset of maladaptive correlates of aging in human beings. This journal is indexed on PubMed Central, MedLine,

\section{Dovepress}

CAS, Scopus and the Elsevier Bibliographic databases. The manuscript management system is completely online and includes a very quick and fair peer-review system, which is all easy to use. Visit http://www.dovepress. com/testimonials.php to read real quotes from published authors. 